\title{
Coupling Analysis on Multiple Interaction of English Mode and Information Technology
}

\author{
1,a Jie Li \\ ${ }^{1}$ College of Foreign Languages, Hebei University, Baoding, Hebei, P.R. China, 071000 \\ a09lijie@gmail.com
}

Keywords: Multiple Interaction, English, Informatization, Mode.

\begin{abstract}
In regard of how to construct an open and efficient teacher education system to realize the teacher's continuous professional development needs, this paper bases on the English major of Hebei University as study materials, to establish Hebei University, itself, multiple interaction on English education mode, conduct analysis on multiple education mode under the informatization background, analyze the coupling calculation mechanism between information technology and English education mode, in order to construct multiple English teacher education mode under the English education background of Hebei University. Through constructing the idea based on optimizing teacher's teaching concept, information quality, teaching method and student's independent study ability, it will improve the high efficient English education training and the professional quality of Hebei University's English teachers, which will further improve the whole English education level.
\end{abstract}

\section{Introduction}

Today, as the graduated student team of the national English major is numerous[1,2] every year plus the overall employment situation of the national college students increasingly becomes severe, however, the employment rate of English major graduated students is high (the contract signing rate is relatively high), but very big challenge still needs to be faced. Therefore, how to serve and construct the English professional talents' training system with special characteristics and the unique property of the economic construction and how to combine social needs and professional location to conduct reform and practice to the original English course system is a big project that English major education of the each college should face.

Specific to the existed gap between theory and practice, deficiency in communication and interaction, shortage in teacher' $\mathrm{s}$ cognition and rethink as well as deficiency in training follow up in Hebei University teachers during the education serve process, based on the education of English teacher as study object, it is put forward to develop teacher' $\mathrm{s}$ rethink ability as the goal using multiple interaction English teacher school-based education mode and use this mode in one university school English teacher school-based education experiment, taking the specific operation as the individual case, in order to discuss its effectiveness improvement in teacher' $s$ rethink ability.

Multiple interactive English teacher school-based education mode under English education background of Hebei University

\section{Multiple interactive analysis on English major}

On the basis of analyzing the involved interaction subject in English teacher education and teaching environment and foreign language teacher school-based education study, it determined the four main factors in multiple interactive English teacher school-based education mode or the main interactive subject including teacher, student, colleague and external researcher. Enlightenment[7] is obtained from "onion ring mode" of Korthagen when construction of multiple interactive English teacher school-based education mode is underway. The biggest factor to influence teacher' s work is "mission", it locates in the inside layer of the "onion ring" with its layers from 
inside to outside self-identification, belief, ability, behavior and environment. The four big interactive subjects in English teacher school-based education environment including teacher, student, colleague and external researcher also consists a four-layer system with layer to layer relationship (see figure 2), its mutual functions construct the main interactive format of the English teacher in the relative complex education and teaching environment, it also plays a critical factor in the process of English teacher school-based education to realize the training and improvement in teacher' s rethink ability goal.

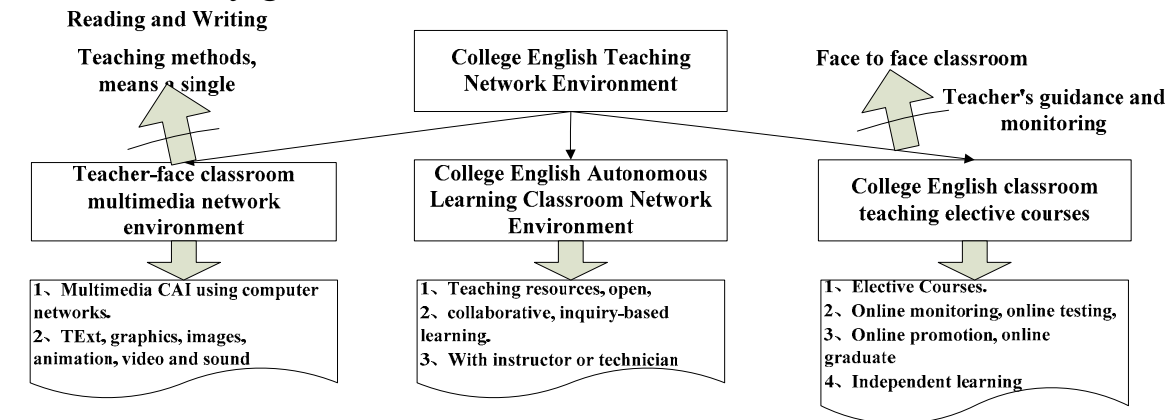

Figure .1:Integrated mode of foreign language teaching course under informative technology

(1) The innermost layer and the core factor is teacher, itself, its interactive format is the interaction between teacher and themselves, namely teacher' $s$ re-think. The outer ring is the student who has the direct relationship with the teacher during teaching practice process, which constructs the second interactive relationship in the multiple interactive mode, namely interaction between the teacher and the student, including teacher' s guidance to the student' $s$ learning and growth, student' $\mathrm{s}$ feedback to the teacher' $\mathrm{s}$ education and teaching work as well as the information, thought, emotion and other communication between the teacher and the student.

(2) The third layer is other teachers in the school, including the teachers responsible for the same subject and the teachers responsible for different subjects. The interactive relationship in this layer includes mutual view and emulate, mutual communication, mutual work and mutual development among the teachers and the colleagues, namely pear coaching, the purpose is to create teacher' s learning community, in order to create school culture which helps the teacher to have continuous professional development.

(3) The outmost layer the interaction between teach and external researcher, including the professional guidance for the teacher from external researcher, and mutual learning and development among researchers.

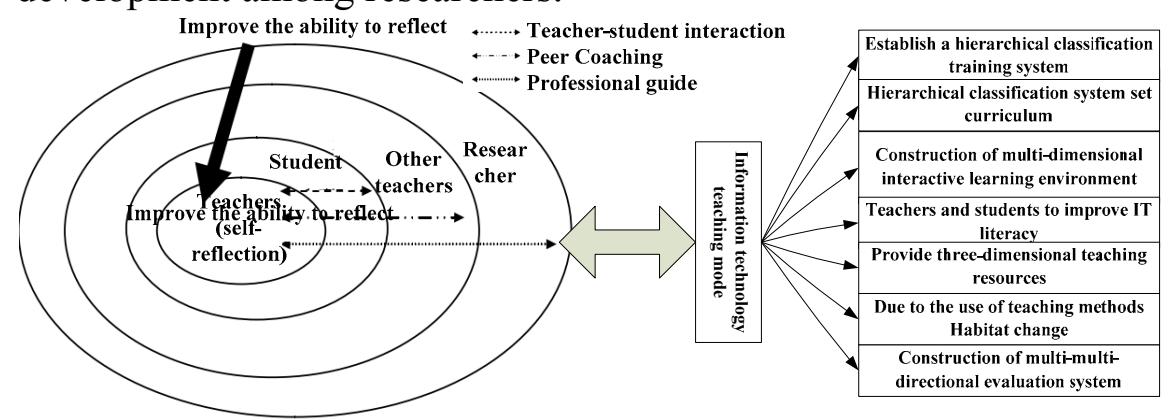

Figure .2: Multiple interactive English teaching mode

\section{Evaluation on intercoupling in multiple interactive mode}

Professional guidance, peer coaching and teacher-student interaction provide external stimulation and information resource for self-rethink. The professional guidance constructs external frame, which accelerates peer coaching and the purpose of teacher-student interaction is to realize teacher' s rethink. However, realization and development of the teacher' $s$ rethink also makes teacher-student interaction, peer coaching and professional guidance more abundant, true and efficient. Teacher-student interaction provides peer coaching the content, peer coaching provides the creation condition for realizing optimum and efficient interaction among teacher-students, and 
peer coaching, teacher-student interaction and self-rethink provide feedback and dynamic needs analysis for the researcher' s professional guidance. The combined three parts also can realize the strong safeguard of continuous development for the teacher after the researcher leaves.

Assume aij, bij and zij represent professional guidance, peer coaching and teacher-student interaction respectively, in order to control multiple points to the English teaching under network environment, it is considered the neutral type coupling and complex dynamic network system with N same points:

$$
\bar{x}_{i}(t)=f\left(x_{i}(t)\right)+\varphi(t) \sum_{N} a_{i j} \Gamma_{x_{j}}(t)+\alpha \sum_{N} b_{i j} \bar{x}_{j}(t)+\beta \sum_{N} c_{i j} \bar{x}_{i}(t)
$$

Where, $x i(t)=\left[x i 1(t), x i 2(t), \ldots, x i n(t) T \in R n\right.$ represents the state vector of No. i point, $f: R^{n} \times R \rightarrow$ $\mathrm{R}^{\mathrm{n}}$ is smooth nonlinearity vector function, $\mathrm{a}$ is the known invariant negative parameter, $\Phi$ (t)represents unknown varying coupling strength, $\Gamma=\operatorname{diag}\left(\gamma_{1}, \ldots, \gamma_{\mathrm{n}}\right)$ is internal coupled matrix. $\mathrm{A}=\left(\right.$ a ij) $\mathrm{N} \times \mathrm{N}$ and $\mathrm{b}=($ bij) $) \mathrm{N} \times \mathrm{N} \in \mathrm{R}^{N^{\times} N}, \mathrm{C}=\left(\mathrm{c}_{i j}\right)^{N^{\times}} \in \mathrm{R}^{N^{\times} N}$ are both external coupled matrix, representing the topological structure of complex network, if $a_{i j}=0\left(b_{i j}=0\right)$, then it represents point $\mathrm{j}$ has influence on the dynamic behavior of $i$, namely from point $\mathrm{j}$ to $\mathrm{i}$, the weight function is $a_{i j}\left(b_{i j}, \mathrm{c}_{\mathrm{ij}}\right)$. The diagonal element of the $\mathrm{A}, \mathrm{B}$ and $\mathrm{C}$ satisfies dissipation condition: : $a i i=-N \sum a i j=1, j=i a i j<0, b i i=-N \sum \mathrm{bij}=1, j=l i b i j<0, c i i=-N \sum \mathrm{cij}=1, j=l i c i j<0, i=1,2, \ldots, N$ 。 .

Coupled matrix $\Gamma$ and $A$ of the network satisfies the following respectively: $\left\||\Gamma \|=\gamma,| a_{i j} \mid \leqslant a, \forall i\right.$, $j=1,2, \ldots, N$ 。

In order to realize each point of the dynamic network at the same time, using self-adaptive strategy to control network $u i(t)$ is described by the controlled network system as:

$$
\bar{x}_{i}(t)=f\left(x_{i}(t)\right)+\varphi(t) \sum_{N} a_{i j} \Gamma_{x_{j}}(t)+\alpha \sum_{N} b_{i j} \bar{x}_{j}(t)+\beta \sum_{N} c_{i j} \bar{x}_{i}(t)+u_{i}(t)
$$

Where, $u_{i}(t)=\left[u_{i 1}(t), u_{i 2}(t), \ldots, u_{i n}(t)\right] \mathrm{T} \in \mathrm{R}^{n}(i=1,2, \ldots, N)$ is self-adaptive which is under designing.

Multiple interaction is an efficient approach to train the rethink ability. The interaction format and content embodies and permeates to the teacher' s rethink, which makes the teacher internalization and learn the knowledge during such process, so it can actually realize their training and improvement of rethink ability. Multiple interaction and the training of the rethink ability construct the continuous positive cycle of the teacher' s professional development. Improvement makes them survey their teaching behavior better and student' s learning effect, more specific to utilize student to improve and adjust teaching to the teaching feedback, so as to improve teacher-student interactive quality.

\section{Optimization demonstration construction of Hebei University English education under informative environment}

\section{Construct optimized teacher's teaching concept, information quality and teaching method as core based teaching frame}

Construct one core bases on multiple modes of optimizing teacher's teaching concept (teaching belief), teacher's information quality and teaching method, to advocate teacher's independence, construct intelligent teacher's development platform, complete teacher's institution training and content informatization's teacher development frame (see figure 3). Under the guidance of ecological theory, according to transition, compatibility and multiple teaching optimization principle to conduct: teaching method transition from traditional to modern, compatible between traditional teaching method and advanced teaching method, so as to establish multiple ecological teaching idea. College English teacher's teaching concept, teacher's information quality 
optimization and establishment of multiple teaching method idea is one of the college English course's teaching optimization cores under the network environment. But, the optimization of such ecological factors needs a modernized teacher development frame as support.

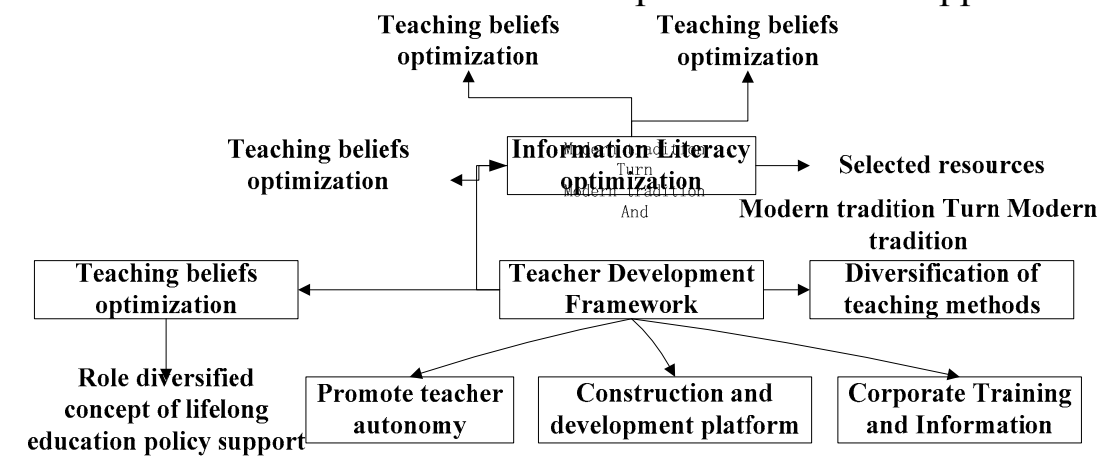

Figure.3: Hebei University English major teacher's development frame

\section{Establish the training frame of student independent study ability}

See figure 4, teacher should change student's learning concept, improve student information quality, train student's learning strategy, independent learning skills and other ability under network environment and strengthen independent learning process monitoring, in order to create a scientific and positive language teaching environment, which makes the training of independent learning ability under network environment as the optimization of college English course teaching core factor. Establish one training frame for the student's independent learning ability, where the optimization of student's self-study concept, information quality, learning motivation, self-study strategy are the core of the frame, the illustrated training and implicit training, emotion adjustment of self-study, external monitoring of the strengthening student independent study, construction of independent study strategy are the powerful support for optimizing the frame.

(1) Student's) self-study concept, study motivation, study strategy, information quality are the important ecological factors in the central ecological system of the college English independent study, they check and balance, none can be dispensed, their coordinating and unifying and joint development are the important content to realize course ecological optimization, and it is the critical point to train the student's independent study ability.

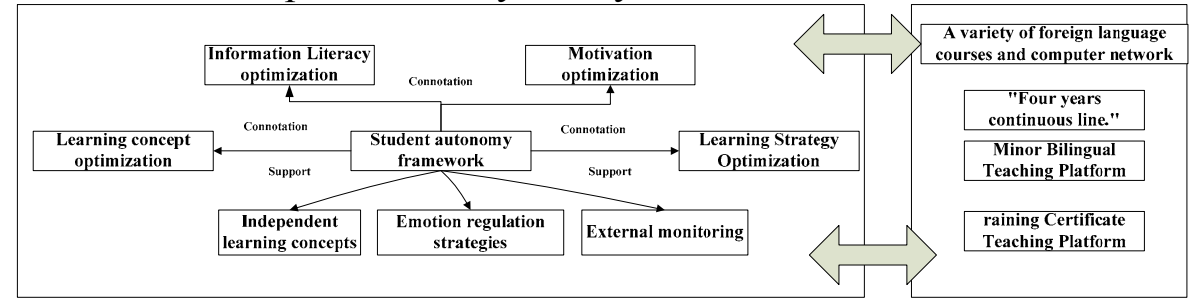

Figure.4: Establish the training diagram of the student' $\mathrm{s}$ independent study

(2) It is needed to guide the student to master emotion adjustment strategy of independent study under network environment to manage self-emotion. And we can control and adjust to the study motivation, confidence, study attitude, attributional pattern, self-efficiency, anxiety and other emotional factors.

(3) Hebei University English major completely uses computer and informative technology to course teaching, and open network and computer based multiple foreign language courses. Fully consider the student's original point, cognitive characteristic, study needs and subjective activity and others, in order to keep the balance between learning subject input and output, realizing deeper understanding and internalization to language knowledge.

(4) Take oral course, cross-cultural communication course and traditional reading, writing, listening and speaking course as the ecological and compatible content, which strengthens student to utilize the knowledge and cross-cultural communication ability to find question, analyze question and solve the question. Carry out college English teaching concept of "No break in four years", rely 
on the three big modules of college English application to improve the teaching platform, double language minor major teaching platform and major certification teaching platform in teaching phase. Construct networked English minor course, broaden student's international view and improve the comprehensive quality of the student. The double background of "Major+English" can improve the student's competition in the future employment.

\section{Example verification and stimulation}

In English learning process, for projection synchronization of varying and complex dynamic network, the network point does not require partial linearity, and scale factor also can be different with each other. Based on stability theory of Lyapunov, for neutral type complex dynamic network, see figure 5, from the evaluation curve of English learning network parameter it can realize the interaction among multiple interactive formats by multiple and specific teacher's education approaches, which forms resultant force accelerating the English teacher's improvement in the criticized rethink ability of self-teaching practice and professional development. It will highlight the mode feature of self-construction, continuously input, cooperation development, individual participation, situation education, emphasis on resultant force, to make good preparation for realization and ability of realizing English teacher's continuous professional development.
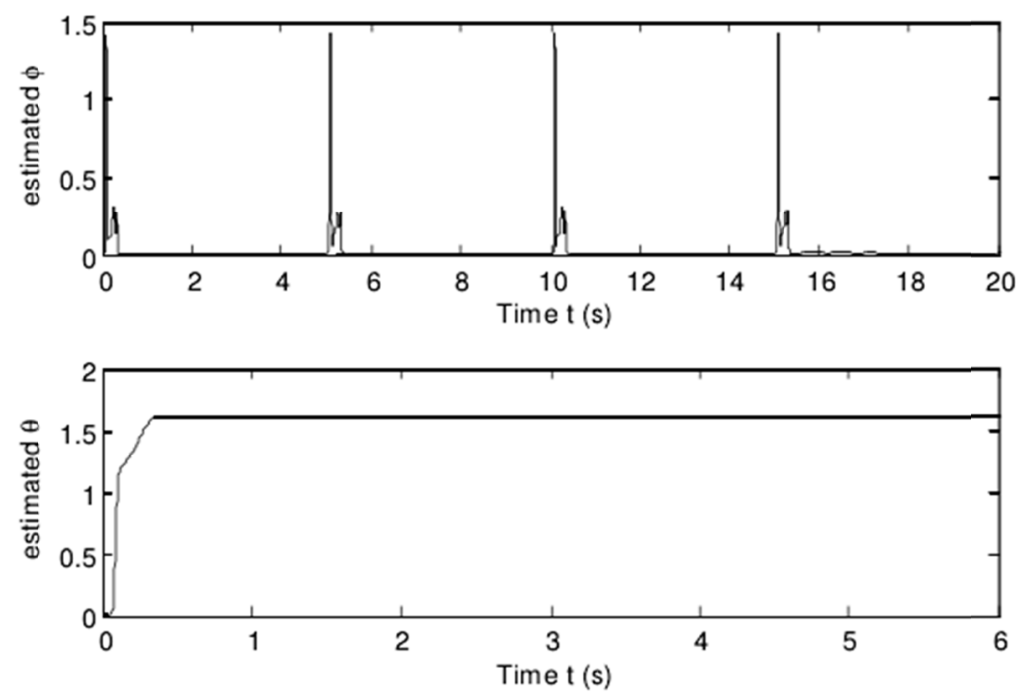

Figure .5: Evaluation curve of English learning network parameter

\section{Conclusion}

In the process of combining informative technology and foreign language teaching, college English teaching goes from traditional course to the college English course under modern network environment. The combination of informative technology and college English course substantially changes the essence of college English teaching. This paper relies on the English major of Hebei University as the study material, conduct multiple interaction interactive English education mode of this major). The multiple interactive English teaching mode advocates that the teacher should base on school' $\mathrm{s}$ education and teaching practice in the English teacher school-based education process, pay close attention to various interaction factors in the teacher' $s$ teaching environment process, improve Hebei University English major' $\mathrm{s}$ education level and quality as well as strengthening the coupling property among informative technology.

\section{References}

[1] Chen Xiangming. Paradox on Qualitative Study Teaching[J]. Education Academic Monthly Publication, 2010,(5):3-4. 
[2] Cui Yunkuo. Survey and Research on China Current Primary and Middle School Teacher Professional Development activity[J]. Global Education Outlook, 2011,(9):25-31.

[3] Cui Yunkuo. School Standard Teacher Professional Development: Frame and Significance[J]. Education Development Research, 2011,(18):67-72.

[4] Dai Ruihua. Teacher Professional Development and School-based Training[M]. Beijing: Science of Education Press, 2011.

[5] Gong Yafu. Establish China Primary and Middle School English Teacher's Knowledge and Ability

System[J]. China Education Magazine,2011, (7):60-65.

[6] Huang Donghua. College English Teacher Cooperation Culture Study- Take Four Colleges in Jiangxi Provinces[J].Heilong Province Higher Education Research,2011, (9):47-49.

[7] Li Banban\&Xu Jinfen. China College English Teacher Rethink Scale Construction[J].China Foreign Language,2011, (4):405-412.

[8] Wang Haixiao. College English Teacher and Teaching Condition Survey and Analysis[J]. Foreign

Language Scope,2009, (4):6-13.

[9] Cai Jigang. Post College English Education Reform Basis and Corresponding Strategy Study[J]. Foreign Language Audio-visual Education, 2010(3): 57-64.

[10] Dai Weidong\&Wang Xuemei. Foreign Language Teacher Professional Development Connotation

and Approach[J]. Foreign Language Audio-visual Education, 2011(6): 8-13.

[11] Gu Shimin. College English Assisted Teaching Mode Study under Virtual Learning Environment-integration Framework Survey of Cooperation Learning and Independent Learning [J]. Foreign Language Audio-visual Education, 2011(6): 59-65. 